Journal of Mathematics and Statistics 4 (3): 138-144, 2008

ISSN 1549-3644

(C) 2008 Science Publications

\title{
Generalization of Differential Operator
}

\author{
Maslina Darus and Rabha W. Ibrahim \\ School of Mathematical Sciences, Faculty of Science and Technology, \\ University Kebangsaan Malaysia, Bangi 43600 Selangor D. Ehsan, Malaysia
}

\begin{abstract}
The main objective of this study was to generalize a differential operator. The generalized differential operator reduced to many known operators studied by various authors. New classes containing this generalized operator were studied and characterization of these classes was obtained. Further, subordination and superordination results involving this operator were studied and obtained the sandwich theorem.
\end{abstract}

Key words: Differential operator, subordination, superordination

\section{INTRODUCTION}

Let $\mathcal{H}$ be the class of functions analytic in $\mathrm{U}$ and $\mathcal{H}[\mathrm{a}, \mathrm{n}]$ be the subclass of $\mathcal{H}$ consisting of functions of the form:

$$
f(z)=a+a_{n} z^{n}+a_{n+1} z^{n+1}+\ldots
$$

Let $\mathrm{A}$ be the subclass of $\mathcal{H}$ consisting of functions of the form:

$f(z)=z+\sum_{n=2}^{\infty} a_{n} z^{n}, \quad z \in U$

Now we introduce a differential operator defines as follows: $\mathrm{D}_{\lambda, \delta}^{\mathrm{k}}: \mathrm{A} \rightarrow \mathrm{A}$ by:

$\mathrm{D}_{\lambda, \delta}^{\mathrm{k}} \mathrm{f}(\mathrm{z})=\mathrm{z}+\sum_{\mathrm{n}=2}^{\infty}[1+(\mathrm{n}-1) \lambda]^{\mathrm{k}} \mathrm{C}(\delta, \mathrm{n}) \mathrm{a}_{\mathrm{n}} \mathrm{z}^{\mathrm{n}}$,

$\mathrm{k} \in \mathbb{N}_{0}, \lambda \geq 0, \delta \geq 0$

Where:

$$
\mathrm{C}(\delta, \mathrm{n})=\left(\begin{array}{c}
\mathrm{n}+\delta-1 \\
\delta
\end{array}\right)=\frac{\Gamma(\mathrm{n}+\delta)}{\Gamma(\mathrm{n}) \Gamma(\delta+1)}
$$

Remark 1: When $\lambda=1, \delta=0$ we get Sălăgean differential operator $^{[8]}, \mathrm{k}=0$ gives Ruscheweyh operator ${ }^{[7]}, \delta=0$ implies Al-Oboudi differential operator of order $(\mathrm{k})^{[1]}$ and when $\lambda=1$ operator (2) reduces to Al-Shaqsi and Darus differential operator of $\operatorname{order}(\mathrm{k})^{[2]}$.
Some of relations for the differential operator (2) are discussed in the next lemma.

Lemma 1: Let $f \in A$. Then:

- $\quad \mathrm{D}_{1,0}^{0} \mathrm{f}(\mathrm{z})=\mathrm{f}(\mathrm{z})$

- $\quad \mathrm{D}_{1,0}^{1} \mathrm{f}(\mathrm{z})=\mathrm{zf} \mathrm{f}^{\prime}(\mathrm{z})$

In the following definitions, new classes of analytic functions containing the differential operator (2) are introduced:

Definition 1: Let $\mathrm{f}(\mathrm{z}) \in \mathrm{A}$.

Then $\mathrm{f}(\mathrm{z}) \in \mathrm{S}_{\lambda, \delta}^{\mathrm{k}}(\mu)$ if and only if:

$$
\mathfrak{R}\left\{\frac{\mathrm{z}\left[\mathrm{D}_{\lambda, \delta}^{\mathrm{k}} \mathrm{f}(\mathrm{z})\right]^{\prime}}{\mathrm{D}_{\lambda, \delta}^{\mathrm{k}} \mathrm{f}(\mathrm{z})}\right\}>\mu, 0 \leq \mu<1, \mathrm{z} \in \mathrm{U}
$$

Definition 2: Let $\mathrm{f}(\mathrm{z}) \in \mathrm{A}$

Then $f(z) \in C_{\lambda, \delta}^{k}(\mu)$ if and only if:

$$
\mathfrak{R}\left\{\frac{\left[\mathrm{z}\left(\mathrm{D}_{\lambda, \delta}^{\mathrm{k}} \mathrm{f}(\mathrm{z})\right)^{\prime}\right]^{\prime}}{\left(\mathrm{D}_{\lambda, \delta}^{\mathrm{k}} \mathrm{f}(\mathrm{z})\right)^{\prime}}\right\}>\mu, 0 \leq \mu<1, \mathrm{z} \in \mathrm{U}
$$

Let $\mathrm{F}$ and $\mathrm{G}$ be analytic functions in the unit disk $U$. The function $F$ is subordinate to $G$, written $F \prec G$ if $\mathrm{G}$ is univalent, $\mathrm{F}(0)=\mathrm{G}(0)$ and $\mathrm{F}(\mathrm{U}) \subset \mathrm{G}(\mathrm{U})$ In general, given two functions $F$ and $G$, which are analytic in $\mathrm{U}$, the function $\mathrm{F}$ is said to be subordinate to $\mathrm{G}$ in $\mathrm{U}$ if there exists a function $\mathrm{h}$, analytic in $\mathrm{U}$ with:

$$
\mathrm{h}(0)=0 \text { and }|\mathrm{h}(\mathrm{z})|<1 \text { for all } \mathrm{z} \in \mathrm{U}
$$

Corresponding Author: Maslina Darus, Faculty of Science and Technology, School of Mathematical Sciences, Universiti Kebangsaan Malaysia, Bangi 43600 Selangor D. Ehsan, Malaysia 
Such that:

$$
\mathrm{F}(\mathrm{z})=\mathrm{G}(\mathrm{h}(\mathrm{z})) \text { for all } \mathrm{z} \in \mathrm{U}
$$

Let $\phi: \mathbb{C}^{2} \rightarrow \mathbb{C}$ and let $h$ be univalent in $\mathrm{U}$. If $\mathrm{p}$ is analytic in $U$ and satisfies the differential subordination $\left.\phi(\mathrm{p}(\mathrm{z})), \mathrm{zp}^{\prime}(\mathrm{z})\right) \prec \mathrm{h}(\mathrm{z})$ then $\mathrm{p}$ is called a solution of the differential subordination. The univalent function $q$ is called a dominant of the solutions of the differential subordination, $\mathrm{p} \prec \mathrm{q}$. If $\mathrm{p}$ and $\left.\phi(\mathrm{p}(\mathrm{z})), \mathrm{zp}^{\prime}(\mathrm{z})\right)$ are univalent in $U$ and satisfy the differential superordination $\mathrm{h}(\mathrm{z}) \prec \phi(\mathrm{p}(\mathrm{z}))$, $\left.\mathrm{zp}^{\prime}(\mathrm{z})\right)$ then $\mathrm{p}$ is called a solution of the differential superordination. An analytic function $\mathrm{q}$ is called subordinant of the solution of the differential superordination if $\mathrm{q} \prec \mathrm{p}$. Let $\Phi$ be an analytic function in a domain containing $\mathrm{f}(\mathrm{U}), \Phi(0)=0$ and $\Phi^{\prime}(0)>0$.

The function $f \in A$ is called $\Phi$-like if:

$$
\mathfrak{R}\left\{\frac{\mathrm{zf}(\mathrm{z})}{\Phi \mathrm{f}(\mathrm{z})}\right\}>0, \mathrm{z} \in \mathrm{U}
$$

This concept was introduced by ${ }^{[3]}$ and established that a function $f \in A$ is univalent if and only if $f$ is $\Phi$ like for some $\Phi$.

Definition 3: Let $\Phi$ be analytic function in a domain containing $\mathrm{f}(\mathrm{U}), \Phi(0)=0, \Phi^{\prime}(0)=1$ and $\Phi(\omega) \neq 0$ for $\omega \in \mathrm{f}(\mathrm{U})-0$. Let $\mathrm{q}(\mathrm{z})$ be a fixed analytic function in $\mathrm{U}$, $\mathrm{q}(0)=1$. The function $\mathrm{f} \in \mathrm{A}$ is called $\Phi$-like with respect to $q$ if:

$$
\left\{\frac{\mathrm{zf}(\mathrm{z})}{\Phi(\mathrm{f}(\mathrm{z}))}\right\} \prec \mathrm{q}(\mathrm{z}), \mathrm{z} \in \mathrm{U}
$$

Definition $4^{[4]}$ : Denote by Q the set of all functions $\mathrm{f}(\mathrm{z})$ that are analytic and injective on $\overline{\mathrm{U}}-\mathrm{E}(\mathrm{f})$ where $\mathrm{E}(\mathrm{f}):=\left\{\zeta \in \partial \mathrm{U}: \lim _{\mathrm{z} \rightarrow \zeta} \mathrm{f}(\mathrm{z})=\infty\right\}$ and are such that $\mathrm{f}^{\prime}(\zeta) \neq 0$ for $\zeta \in \partial \mathrm{U}-\mathrm{E}(\mathrm{f})$.

Lemma $2^{[5]}$ : Let $q(\mathrm{z})$ be univalent in the unit disk $\mathrm{U}$ and $\theta$ and $\phi$ be analytic in a domain D containing $q(U)$ with $\phi(\omega) \neq 0$ when $\omega \in \mathrm{q}(\mathrm{U})$. Set $\mathrm{Q}(\mathrm{z}):=\mathrm{zq}^{\prime}(\mathrm{z}) \phi(\mathrm{q}(\mathrm{z}))$, $\mathrm{h}(\mathrm{z}):=\theta(\mathrm{q}(\mathrm{z}))+\mathrm{Q}(\mathrm{z})$. Suppose that:

- $\mathrm{Q}(\mathrm{z})$ is starlike univalent in $\mathrm{U}$

- $\mathfrak{R} \frac{\mathrm{zh}^{\prime}(\mathrm{z})}{\Phi(\mathrm{z})}>0$ for $\mathrm{z} \in \mathrm{U}$

If:

$$
\theta(\mathrm{p}(\mathrm{z}))+\mathrm{zp}^{\prime}(\mathrm{z}) \phi(\mathrm{p}(\mathrm{z})) \prec \theta(\mathrm{q}(\mathrm{z}))+\mathrm{zq}^{\prime}(\mathrm{z}) \phi(\mathrm{q}(\mathrm{z}))
$$

Then:

$$
\mathrm{p}(\mathrm{z}) \prec \mathrm{q}(\mathrm{z})
$$

and $\mathrm{q}(\mathrm{z})$ is the best dominant.

Lemma $3^{[6]}$ : Let $\mathrm{q}(\mathrm{z})$ be convex univalent in the unit disk $U$ and $\vartheta$ and $\varphi$ be analytic in a domain $D$ containing $q(U)$. Suppose that:

- $\quad \mathrm{zq}^{\prime}(\mathrm{z}) \varphi(\mathrm{q}(\mathrm{z}))$ is star like univalent in $\mathrm{U}$

- $\mathfrak{R}\left\{\frac{\vartheta^{\prime}(\mathrm{q}(\mathrm{z}))}{\varphi(\mathrm{q}(\mathrm{z}))}\right\}>0$ for $\mathrm{z} \in \mathrm{U}$

If $\mathrm{p}(\mathrm{z}) \in \mathcal{H}[\mathrm{q}(0), 1] 1 \mathrm{Q}$, with $\mathrm{p}(\mathrm{U}) \subseteq \mathrm{D}$ and $\vartheta$ $(\mathrm{p}(\mathrm{z}))+\mathrm{zp}^{\prime}(\mathrm{z}) \varphi(\mathrm{z})$ is univalent in $\mathrm{U}$ and $\vartheta(\mathrm{q}(\mathrm{z}))+\mathrm{zq}^{\prime}(\mathrm{z})$ $\varphi(\mathrm{q}(\mathrm{z})) \prec \vartheta(\mathrm{p}(\mathrm{z}))+\mathrm{zp}^{\prime}(\mathrm{z}) \varphi(\mathrm{p}(\mathrm{z}))$ then $\mathrm{q}(\mathrm{z}) \prec \mathrm{p}(\mathrm{z})$ and $\mathrm{q}(\mathrm{z})$ is the best subordinant.

\section{MATERIAL AND MATHODS}

General properties of $\mathbf{D}_{\lambda, \delta}^{\mathbf{k}}$ : In this research we study the characterization properties for the function $\mathrm{f} \in \mathrm{A}$ to belong to the classes $S_{\lambda, \delta}^{k}(\mu)$ and $C_{\lambda, \delta}^{k}(\mu)$ by obtaining the coefficient bounds.

Theorem 1: Let the function $\mathrm{f} \in \mathrm{A}$. If

$$
\sum_{n=2}^{\infty}(n-\mu)[1+(n-1) \lambda]^{k} C(\delta, n)\left|a_{n}\right| \leq 1-\mu
$$

$0 \leq \mu<1$

Then $f \in S_{\lambda, \delta}^{k}(\mu)$. The result (3) is sharp.

Proof: Suppose that (3) holds. Since:

$$
\begin{aligned}
1-\mu & \geq \sum_{\mathrm{n}=2}^{\infty}(\mathrm{n}-\mu)[1+(\mathrm{n}-1) \lambda]^{\mathrm{k}} \mathrm{C}(\delta, \mathrm{n})\left|\mathrm{a}_{\mathrm{n}}\right| \\
& \geq \sum_{\mathrm{n}=2}^{\infty} \mu[1+(\mathrm{n}-1) \lambda]^{\mathrm{k}} \mathrm{C}(\delta, \mathrm{n})\left|\mathrm{a}_{\mathrm{n}}\right| \\
& -\sum_{\mathrm{n}=2}^{\infty} \mathrm{n}[1+(\mathrm{n}-1) \lambda]^{\mathrm{k}} \mathrm{C}(\delta, \mathrm{n})\left|\mathrm{a}_{\mathrm{n}}\right|
\end{aligned}
$$

then this implies that:

$$
\frac{1+\sum_{n=2}^{\infty} n[1+(n-1) \lambda]^{k} C(\delta, n)\left|a_{n}\right|}{1+\sum_{n=2}^{\infty}[1+(n-1) \lambda]^{k} C(\delta, n)\left|a_{n}\right|}>\mu
$$

Hence: 


$$
\mathfrak{R}\left\{\frac{\mathrm{z}\left[\mathrm{D}_{\lambda, \delta}^{\mathrm{k}} \mathrm{f}(\mathrm{z})\right]^{\prime}}{\mathrm{D}_{\lambda, \delta}^{\mathrm{k}} \mathrm{f}(\mathrm{z})}\right\}>\mu
$$

We also note that the assertion (3) is sharp and the extremal function is given by:

$$
f(z)=z+\sum_{n=2}^{\infty} \frac{(1-\mu)}{(n-\mu)[1+(n-1) \lambda]^{k} C(\delta, n)} z^{n}
$$

\section{Corollary 1: If}

$$
\left|\mathrm{a}_{\mathrm{n}}\right| \leq \frac{(1-\mu)}{(\mathrm{n}-\mu)[1+(\mathrm{n}-1) \lambda]^{\mathrm{k}} \mathrm{C}(\delta, \mathrm{n})}, \forall \mathrm{n} \geq 2
$$

Then the function $\mathrm{f}$ belongs to the class $S_{\lambda, \delta}^{\mathrm{k}}(\mu)$.

Corollary 2: If for $\delta=\mu=0$ and $\lambda=1$ :

$$
\left|\mathrm{a}_{\mathrm{n}}\right| \leq \frac{1}{\mathrm{n}^{\mathrm{k}}}, \forall \mathrm{n} \geq 2, \mathrm{k} \in \mathbb{N}_{0}
$$

Then the function $\mathrm{f}$ belongs to the class $S_{\lambda, \delta}^{\mathrm{k}}(\mu)$. results:

In the same way we can verify the following

Theorem 2: Let the function $f \in A$. If

$\sum_{n=2}^{\infty} n(n-\mu)[1+(n-1) \lambda]^{k} C(\delta, n)\left|a_{n}\right| \leq 1-\mu, 0 \leq \mu<1$

Then $f(z) \in C_{\lambda, \delta}^{k}(\mu)$. The result (6) is sharp.

\section{Corollary 3: If}

$$
\left|a_{n}\right| \leq \frac{(1-\mu)}{n(n-\mu)[1+(n-1) \lambda]^{k} C(\delta, n)}, \forall n \geq 2
$$

Then the function $\mathrm{f}$ belongs to the class $\mathrm{C}_{\lambda, \delta}^{\mathrm{k}}(\mu)$.

Also we have the following inclusion results:

Theorem 3: Let the assumption of theorem 1 holds and $0 \leq \mu_{1} \leq \mu_{2}<1$. Then $S_{\lambda, \delta}^{k}\left(\mu_{1}\right) \supseteq S_{\lambda, \delta}^{k}\left(\mu_{2}\right)$.

Proof: By theorem 1.

Theorem 4: Let the assumption of theorem 2 holds and $0 \leq \mu_{1} \leq \mu_{2}<1$. Then $\mathrm{C}_{\lambda, \delta}^{\mathrm{k}}\left(\mu_{1}\right) \supseteq \mathrm{C}_{\lambda, \delta}^{\mathrm{k}}\left(\mu_{2}\right)$.
Proof: By theorem 2.

Theorem 5: Let the assumption of theorem 1 holds and $0 \leq \lambda_{1} \leq \lambda_{2}<1$. Then $\mathrm{S}_{\lambda_{1}, \delta}^{\mathrm{k}}(\mu) \supseteq \mathrm{S}_{\lambda_{2}, \delta}^{\mathrm{k}}(\mu)$.

Proof: By theorem 1.

Theorem 6: Let the assumption of theorem 2 holds and $0 \leq \lambda_{1} \leq \lambda_{2}$. Then $C_{\lambda_{1}, \delta}^{k}(\mu) \supseteq C_{\lambda_{2}, \delta}^{k}(\mu)$.

Proof: By theorem 2.

Moreover, we introduce the following distortion theorems.

Theorem 7: Let the function $f \in A$ and (3) holds. Then for $\mathrm{z} \in \mathrm{U}$ and $0 \leq \mu<1$ :

$$
\left|D_{\lambda, \delta}^{k} f(z)\right| \geq|z|-\frac{1-\mu}{2-\mu}
$$

and

$$
\left|D_{\lambda, \delta}^{k} f(z)\right| \leq|z|+\frac{1-\mu}{2-\mu}
$$

Proof: By using theorem 1, one can verify that:

$$
\begin{aligned}
& (2-\mu) \sum_{n=2}^{\infty}[1+(n-1) \lambda]^{k} C(\delta, n)\left|a_{n}\right| \leq \\
& \sum_{n=2}^{\infty}(n-\mu)[1+(n-1) \lambda]^{k} C(\delta, n)\left|a_{n}\right| \leq 1-\mu
\end{aligned}
$$

Then:

$$
\sum_{n=2}^{\infty}[1+(n-1) \lambda]^{k} C(\delta, n)\left|a_{n}\right| \leq \frac{1-\mu}{2-\mu}
$$

Thus we obtain:

$$
\begin{aligned}
\left|D_{\lambda, \delta}^{\mathrm{k}} \mathrm{f}(\mathrm{z})\right| \leq|\mathrm{z}|+\sum_{\mathrm{n}=2}^{\infty}(\mathrm{n}-\mu)[1+(\mathrm{n}-1) \lambda]^{\mathrm{k}} \mathrm{C}(\delta, \mathrm{n})\left|\mathrm{a}_{\mathrm{n}}\right||\mathrm{z}|^{\mathrm{n}} \\
\quad \leq|\mathrm{z}|+\left[\frac{1-\mu}{2-\mu}\right]|\mathrm{z}|^{2}
\end{aligned}
$$

The other assertion can be proved as follows: 


$$
\begin{aligned}
\left|D_{\lambda, \delta}^{k} f(z)\right| & =\mid z+\sum_{n=2}^{\infty}(n-\mu)[1+(n-1) \lambda]^{k} C(\delta, n) a_{n} z^{n} \\
& \geq|z|-\sum_{n=2}^{\infty}(n-\mu)\left[1+(n-1) \lambda C(\delta, n)\left|a_{n}\right||z|^{n}\right. \\
& \geq|z|-\left[\frac{1-\mu}{2-\mu}\right]|z|^{2}
\end{aligned}
$$

This completes the proof.

In the same way we can get the following results.

Theorem 8: Let the functions $\mathrm{f} \in \mathrm{A}$ and (6) holds. Then for $\mathrm{z} \in \mathrm{U}$ and $0 \leq \mu<1$ :

$$
\left|D_{\lambda, \delta}^{k} f(z)\right| \geq|z|-\frac{(1-\mu)}{2(2-\mu)}|z|^{2}
$$

and

$$
\left|D_{\lambda, \delta}^{k} f(z)\right| \leq|z|+\frac{(1-\mu)}{2(2-\mu)}|z|^{2}
$$

Also, we have the following distortion results.

Theorem 9: Let the function $\mathrm{f} \in \mathrm{A}$ and (3) holds. Then for $(n-\mu)[1+(n-1) \lambda]^{k} C(\delta, n) \geq 1$ and $0 \leq \mu<1$, we have:

$$
|f(z)| \geq|z|-(1-\mu)|z|^{2}
$$

and

$$
|f(z)| \leq|z|+(1-\mu)|z|^{2}
$$

Proof: In virtue of theorem 1, we have:

$$
\sum_{n=2}^{\infty}\left|a_{n}\right| \leq \sum_{n=2}^{\infty}(n-\mu)[1+(n-1) \lambda]^{k} C(\delta, n)\left|a_{n}\right| \leq 1-\mu
$$

Then:

$$
\sum_{n=2}^{\infty}\left|a_{n}\right| \leq(1-\mu)
$$

Thus we obtain:

$$
\begin{aligned}
|f(z)| & \left|z+\sum_{n=2}^{\infty} a_{n} z^{n}\right| \\
& \leq|z|+\sum_{n=2}^{\infty}\left|a_{n}\right||z|^{2} \\
& \leq|z|+(1-\mu)|z|^{2}
\end{aligned}
$$

The other assertion can be proved as follows:

$$
\begin{aligned}
|f(z)| & =\left|z+\sum_{n=2}^{\infty} a_{n} z^{n}\right| \\
& \geq|z|-\sum_{n=2}^{\infty}\left|a_{n}\right||z|^{2} \\
& \geq|z|-(1-\mu)|z|^{2}
\end{aligned}
$$

This completes the proof.

In the same way we can get the following results.

Theorem 10: Let the function $f \in A$ and (6) holds. Then for $(\mathrm{n}-\mu)[1+(\mathrm{n}-1) \lambda]^{\mathrm{k}} \mathrm{C}(\delta, \mathrm{n}) \geq 1$ and $0 \leq \mu<1$ :

$$
|\mathrm{f}(\mathrm{z})| \geq|\mathrm{z}|-\frac{(1-\mu)}{2}|\mathrm{z}|^{2}
$$

and

$$
|f(z)| \leq|z|+\frac{(1-\mu)}{2}|z|^{2}
$$

\section{RESULTS}

By making use of lemmas 2 and 3, we prove the following subordination and superordination results involving the differential operator (2).

Theorem 11: Let $q(z) \neq 0$ be univalent in $U$ such that $\frac{\mathrm{zq}^{\prime}(\mathrm{z})}{\mathrm{q}(\mathrm{z})}$ is starlike univalent in $\mathrm{U}$ and:

$\Re\left\{1-\frac{\alpha}{\gamma \mathrm{q}(\mathrm{z})}+\frac{\mathrm{zq} \mathrm{q}^{\prime \prime}(\mathrm{z})}{\mathrm{q}^{\prime}(\mathrm{z})}-\frac{\mathrm{zq}(\mathrm{z})}{\mathrm{q}(\mathrm{z})}\right\}>0, \alpha, \gamma \in \div, \gamma \neq 0$

If $\mathrm{f} \in \mathrm{A}$ satisfies the subordination:

$$
\begin{aligned}
& \alpha\left(\frac{\mathrm{z}\left[\mathrm{D}_{\lambda, \delta}^{\mathrm{k}} \mathrm{f}(\mathrm{z})\right]^{\prime}}{\mathrm{D}_{\lambda, \delta}^{\mathrm{k}} \mathrm{f}(\mathrm{z})}\right)^{-1}+\gamma\left\{1+\frac{\mathrm{z}\left[\mathrm{D}_{\lambda, \delta}^{\mathrm{k}} \mathrm{f}(\mathrm{z})\right]^{\prime \prime}}{\mathrm{D}_{\lambda, \delta}^{\mathrm{k}} \mathrm{f}(\mathrm{z})^{\prime}}-\frac{\mathrm{z} \Phi^{\prime}\left[\mathrm{D}_{\lambda, \delta}^{\mathrm{k}} \mathrm{f}(\mathrm{z})\right]}{\Phi\left[\mathrm{D}_{\lambda, \delta}^{\mathrm{k}} \mathrm{f}(\mathrm{z})\right]}\right\} \\
& \prec \frac{\alpha}{\mathrm{q}(\mathrm{z})}+\frac{\gamma \mathrm{zq}(\mathrm{z})}{\mathrm{q}(\mathrm{z})}, \mathrm{z} \neq 0
\end{aligned}
$$

Then:

$$
\left.\frac{\mathrm{z}\left[\mathrm{D}_{\lambda, \delta}^{\mathrm{k}} \mathrm{f}(\mathrm{z})\right]^{\prime}}{\Phi\left[\mathrm{D}_{\lambda, \delta}^{\mathrm{k}} \mathrm{f}(\mathrm{z})\right]^{\prime}}\right\} \prec \mathrm{q}(\mathrm{z})
$$

and $\mathrm{q}(\mathrm{z})$ is the best dominant. 
Proof: Our aim is to apply lemma 2. Setting:

$$
\mathrm{p}(\mathrm{z}):=\frac{\mathrm{z}\left[\mathrm{D}_{\lambda, \delta}^{\mathrm{k}} \mathrm{f}(\mathrm{z})\right]^{\prime}}{\Phi\left[\mathrm{D}_{\lambda, \delta}^{\mathrm{k}} \mathrm{f}(\mathrm{z})\right]}
$$

By computation shows that:

$$
\frac{\mathrm{zp}^{\prime}(\mathrm{z})}{\mathrm{p}(\mathrm{z})}=1+\frac{\mathrm{z}\left[\mathrm{D}_{\lambda, \delta}^{\mathrm{k}} \mathrm{f}(\mathrm{z})\right]^{\prime \prime}}{\left[\mathrm{D}_{\lambda, \delta}^{\mathrm{k}} \mathrm{f}(\mathrm{z})\right]^{\prime}}-\frac{z \Phi^{\prime}\left[\mathrm{D}_{\lambda, \delta}^{\mathrm{k}} \mathrm{f}(\mathrm{z})\right]}{\Phi\left[\mathrm{D}_{\lambda, \delta}^{\mathrm{k}} \mathrm{f}(\mathrm{z})\right]}
$$

which yields the following subordination:

$$
\frac{\alpha}{\mathrm{p}(\mathrm{z})}+\frac{\gamma \mathrm{zp} \mathrm{p}^{\prime}(\mathrm{z})}{\mathrm{p}(\mathrm{z})} \prec \frac{\alpha}{\mathrm{q}(\mathrm{z})}+\frac{\gamma \mathrm{zq} \mathrm{\prime}^{\prime}(\mathrm{z})}{\mathrm{q}(\mathrm{z})}, \alpha, \gamma \in \mathbb{C}
$$

By setting:

$$
\theta(\omega):=\frac{\alpha}{\omega} \text { and } \phi(\omega):=\frac{\gamma}{\omega}, \gamma \neq 0
$$

It can be observed that $\theta(\omega), \phi(\omega)$ are analytic in $\mathbb{C} /\{0\}$ and that $\phi(\omega) \neq 0$ when $\omega \in \mathbb{C} /\{0\}$.

Also by letting:

$$
\mathrm{Q}(\mathrm{z})=\mathrm{zq} \mathrm{q}^{\prime}(\mathrm{z}) \phi(\mathrm{q}(\mathrm{z}))=\gamma \mathrm{z} \frac{\mathrm{q}^{\prime}(\mathrm{z})}{\mathrm{q}(\mathrm{z})}
$$

and

$$
\mathrm{h}(\mathrm{z})=\theta(\mathrm{q}(\mathrm{z}))+\mathrm{Q}(\mathrm{z})=\frac{\alpha}{\mathrm{q}(\mathrm{z})}+\gamma \mathrm{z} \frac{\mathrm{q}^{\prime}(\mathrm{z})}{\mathrm{q}(\mathrm{z})}
$$

we find that $\mathrm{Q}(\mathrm{z})$ is starlike univalent in $\mathrm{U}$ and that:

$$
\mathfrak{R}\left\{\frac{\mathrm{zh}(\mathrm{z})}{\mathrm{Q}(\mathrm{z})}\right\}=\Re\left\{1-\frac{\alpha}{\gamma \mathrm{q}(\mathrm{z})}+\frac{\mathrm{zq}^{\prime \prime}(\mathrm{z})}{\mathrm{q}^{\prime}(\mathrm{z})}-\frac{\mathrm{zq}^{\prime}(\mathrm{z})}{\mathrm{q}(\mathrm{z})}\right\}>0
$$

For $\alpha, \gamma \in \mathbb{C}, \gamma \neq 0$.

Then the relation (9) follows by an application of lemma 2.

Corollary 4: Let the assumptions of theorem 11 hold. Then the subordination:

$$
\begin{aligned}
& \alpha\left(\frac{\mathrm{z}\left[\mathrm{D}_{\lambda, \delta}^{\mathrm{k}} \mathrm{f}(\mathrm{z})\right]^{\prime}}{\mathrm{D}_{\lambda, \delta}^{\mathrm{k}} \mathrm{f}(\mathrm{z})}\right)^{-1}+\gamma\left\{1+\frac{\mathrm{z}\left[\mathrm{D}_{\lambda, \mathrm{\delta}}^{\mathrm{k}} \mathrm{f}(\mathrm{z})\right]^{\prime \prime}}{\mathrm{D}_{\lambda, \delta}^{\mathrm{k}} \mathrm{f}(\mathrm{z})^{\prime}}-\frac{\mathrm{z}\left[\mathrm{D}_{\lambda, \delta}^{\mathrm{k}} \mathrm{f}(\mathrm{z})\right]^{\prime}}{\left[\mathrm{D}_{\lambda, \delta}^{\mathrm{k}} \mathrm{f}(\mathrm{z})\right]^{\prime}}\right\} \\
& \prec \frac{\alpha}{\mathrm{q}(\mathrm{z})}+\frac{\gamma \mathrm{zq}(\mathrm{z})}{\mathrm{q}(\mathrm{z})}
\end{aligned}
$$

Implies:

$$
\frac{\mathrm{z}\left[\mathrm{D}_{\lambda, \delta}^{\mathrm{k}} \mathrm{f}(\mathrm{z})\right]^{\prime}}{\left[\mathrm{D}_{\lambda, \delta}^{\mathrm{k}} \mathrm{f}(\mathrm{z})\right]} \prec \mathrm{q}(\mathrm{z})
$$

and $\mathrm{q}(\mathrm{z})$ is the best dominant.

Proof: By letting $\Phi(\omega):=\omega$.

Corollary 5: If $f \in A$ and assume that (8) holds then:

$$
1+\frac{z\left[D_{\lambda, \delta}^{k} f(z)\right]^{\prime \prime}}{\left[D_{\lambda, \delta}^{k} f(z)\right]^{\prime}}-\frac{z\left[D_{\lambda, \delta}^{k} f(z)\right]^{\prime}}{\left[D_{\lambda, \delta}^{k} f(z)\right]} \prec \frac{(A-B) z}{(1+A z)(1+B z)}
$$

Implies:

$$
\frac{\mathrm{z}\left[\mathrm{D}_{\lambda, \delta}^{\mathrm{k}} \mathrm{f}(\mathrm{z})\right]}{\left[\mathrm{D}_{\lambda, \delta}^{\mathrm{k}} \mathrm{f}(\mathrm{z})\right]} \prec \frac{1+\mathrm{Az}}{1+\mathrm{Bz}},-1 \leq \mathrm{B}<\mathrm{A} \leq 1
$$

and $\frac{1+\mathrm{Az}}{1+\mathrm{Bz}}$ is the best dominant.

Proof: By setting $\Phi(\omega)$ : $=\omega, \alpha=0, \gamma=1$ and $\mathrm{q}(\mathrm{z}):=\frac{1+\mathrm{Az}}{1+\mathrm{Bz}}$ where $-1 \leq \mathrm{B}<\mathrm{A} \leq 1$.

Corollary 6: If $f \in A$ and assume that (8) holds then:

$$
1+\frac{z\left[D_{\lambda, \delta}^{k} f(z)\right]^{\prime \prime}}{\left[D_{\lambda, \delta}^{k} f(z)\right]^{\prime}}-\frac{z\left[D_{\lambda, \delta}^{k} f(z)\right]^{\prime}}{\left[D_{\lambda, \delta}^{k} f(z)\right]} \prec \frac{2 z}{1-z^{2}}
$$

Implies:

$$
\frac{\mathrm{z}\left[\mathrm{D}_{\lambda, \delta}^{\mathrm{k}} \mathrm{f}(\mathrm{z})\right]^{\prime}}{\mathrm{D}_{\lambda, \delta}^{\mathrm{k}} \mathrm{f}(\mathrm{z})} \prec \frac{1+\mathrm{z}}{1-\mathrm{z}}
$$

and $\frac{1+z}{1-z}$ is the best dominant.

Proof: By setting $\Phi(\omega):=\omega, \alpha=0, \gamma=1$ and $\mathrm{q}(\mathrm{z}):=$ $\frac{1+\mathrm{z}}{1-\mathrm{z}}$.

Corollary 7: If $f \in A$ and assume that (8) holds then:

Implies:

$$
1+\frac{z\left[D_{\lambda, \delta}^{k} f(z)\right]^{\prime \prime}}{\left[D_{\lambda, \delta}^{k} f(z)\right]^{\prime}}-\frac{z\left[D_{\lambda, \delta}^{k} f(z)\right]^{\prime}}{\left[D_{\lambda, \delta}^{k} f(z)\right]} \prec A z
$$




$$
\frac{z\left[D_{\lambda, \delta}^{k} f(z)\right]^{\prime}}{D_{\lambda, \delta}^{k} f(z)} \prec e^{A z}
$$

and $\mathrm{e}^{\mathrm{Az}}$ is the best dominant.

Proof: By setting $\Phi(\omega):=\omega, \quad \alpha=0, \quad \gamma=1$ and $\mathrm{q}(\mathrm{z}):=\mathrm{e}^{\mathrm{Az}},|\mathrm{A}|<\pi$.

Theorem 12: Let $\mathrm{q}(\mathrm{z}) \neq 0$ be convex univalent in the unit disk U. Suppose that:

$\mathfrak{R}\left\{\frac{-\alpha}{\gamma \mathrm{q}(\mathrm{z})}\right\}>0, \alpha, \gamma \in \mathbb{C}$ for $\mathrm{z} \in \mathrm{U}$

and $\frac{\mathrm{zq}^{\prime}(\mathrm{z})}{\mathrm{q}(\mathrm{z})}$ is starlike univalent in $\mathrm{U}$.

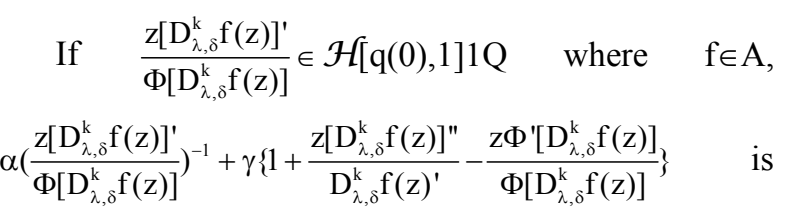

univalent in $\mathrm{U}$ and the subordination:

$$
\begin{aligned}
& \frac{\alpha}{\mathrm{q}(\mathrm{z})}+\frac{\gamma \mathrm{zq}(\mathrm{z})}{\mathrm{q}(\mathrm{z})} \\
& \prec \alpha\left(\frac{\mathrm{z}\left[\mathrm{D}_{\lambda, \delta}^{\mathrm{k}} \mathrm{f}(\mathrm{z})\right]^{\prime}}{\Phi\left[\mathrm{D}_{\lambda, \delta}^{\mathrm{k}} \mathrm{f}(\mathrm{z})\right]^{-1}}\right)^{-1}+\gamma\left\{1+\frac{\mathrm{z}\left[\mathrm{D}_{\lambda, \delta}^{\mathrm{k}} \mathrm{f}(\mathrm{z})\right]^{\prime \prime}}{\mathrm{D}_{\lambda, \delta}^{\mathrm{k}} \mathrm{f}(\mathrm{z})^{\prime}}-\frac{\mathrm{z}^{\prime}\left[\mathrm{D}_{\lambda, \delta}^{\mathrm{k}} \mathrm{f}(\mathrm{z})\right]}{\Phi\left[\mathrm{D}_{\lambda, \delta}^{\mathrm{k}} \mathrm{f}(\mathrm{z})\right]}\right\}
\end{aligned}
$$

holds, then:

$$
q(z) \prec \frac{z\left[D_{\lambda, \delta}^{k} f(z)\right]^{\prime}}{\Phi\left[D_{\lambda, \delta}^{k} f(z)\right]}
$$

and $\mathrm{q}$ is the best subordinant.

Proof: Our aim is to apply lemma 3. Setting:

$$
\mathrm{p}(\mathrm{z}):=\frac{z\left[\mathrm{D}_{\lambda, \delta}^{\mathrm{k}} \mathrm{f}(\mathrm{z})\right]^{\prime}}{\Phi\left[\mathrm{D}_{\lambda, \delta}^{\mathrm{k}} \mathrm{f}(\mathrm{z})\right]}
$$

By a simple computation, shows that:

$$
\frac{z p^{\prime}(z)}{p(z)}=1+\frac{z\left[D_{\lambda, \delta}^{k} f(z)\right]^{\prime \prime}}{\left[D_{\lambda, \delta}^{k} f(z)\right]^{\prime}}-\frac{z \Phi^{\prime}\left[D_{\lambda, \delta}^{k} f(z)\right]}{\Phi\left[D_{\lambda, \delta}^{k} f(z)\right]}
$$

Which yields the following subordination:

$$
\frac{\alpha}{\mathrm{p}(\mathrm{z})}+\frac{\gamma \mathrm{zq}(\mathrm{z})}{\mathrm{q}(\mathrm{z})} \prec \frac{\alpha}{\mathrm{p}(\mathrm{z})}+\frac{\gamma \mathrm{zp}(\mathrm{z})}{\mathrm{p}(\mathrm{z})}, \alpha, \gamma \in \mathbb{C}
$$

By setting:

$$
\vartheta(\omega):=\frac{\alpha}{\omega} \text { and } \varphi(\omega):=\frac{\gamma}{\omega}, \gamma \neq 0
$$

it can be easily observed that $\vartheta(\omega), \varphi(\omega)$ are analytic in $\mathbb{C} \backslash\{0\}$ and that $\varphi(\omega) \neq 0$ when $\omega \in \mathbb{C} \backslash\{0\}$.

Also, we obtain:

$$
\mathfrak{R}\left\{\frac{\vartheta^{\prime}(\mathrm{q}(\mathrm{z}))}{\varphi(\mathrm{q}(\mathrm{z}))}\right\}=\Re \frac{-\alpha}{\gamma \mathrm{q}(\mathrm{z})}>0
$$

Then (12) follows by an application of lemma 3.

By combining theorems 11 and 12 , we get the following sandwich theorems:

Theorem 13: Let $\mathrm{q}_{1}(\mathrm{z}) \neq 0, \mathrm{q}_{2}(\mathrm{z}) \neq 0$ be convex univalent in the unit disk U satisfy (11) and (8) respectively. Suppose that $\frac{z_{\mathrm{i}}^{\prime}(\mathrm{z})}{\mathrm{q}_{\mathrm{i}}(\mathrm{z})}, \mathrm{i}=1,2$ is starlike univalent in $\mathrm{U}$. If $\mathrm{f} \in \mathrm{A}$ and:

$$
\alpha\left(\frac{\mathrm{z}\left[\mathrm{D}_{\lambda, \delta}^{\mathrm{k}} \mathrm{f}(\mathrm{z})\right]^{\prime}}{\Phi\left[\mathrm{D}_{\lambda, \delta}^{\mathrm{k}} \mathrm{f}(\mathrm{z})\right]^{-1}}+\gamma\left\{1+\frac{\mathrm{z}\left[\mathrm{D}_{\lambda, \delta}^{\mathrm{k}} \mathrm{f}(\mathrm{z})\right]^{\prime \prime}}{\mathrm{D}_{\lambda, \delta}^{\mathrm{k}} \mathrm{f}(\mathrm{z})^{\prime}}-\frac{\mathrm{z} \Phi^{\prime}\left[\mathrm{D}_{\lambda, \delta}^{\mathrm{k}} \mathrm{f}(\mathrm{z})\right]}{\Phi\left[\mathrm{D}_{\lambda, \delta}^{\mathrm{k}} \mathrm{f}(\mathrm{z})\right]}\right\}\right.
$$

is univalent in $\mathrm{U}$ and the subordination:

$$
\begin{aligned}
& \frac{\alpha}{\mathrm{q}_{1}(\mathrm{z})}+\frac{\gamma \mathrm{zq}_{1}^{\prime}(\mathrm{z})}{\mathrm{q}_{1}(\mathrm{z})} \\
& \prec \alpha\left(\frac{\mathrm{z}\left[\mathrm{D}_{\lambda, \delta}^{\mathrm{k}} \mathrm{f}(\mathrm{z})\right]^{\prime}}{\Phi\left[\mathrm{D}_{\lambda, \delta}^{\mathrm{k}} \mathrm{f}(\mathrm{z})\right]^{-1}}+\gamma\left\{1+\frac{\mathrm{z}\left[\mathrm{D}_{\lambda, \delta}^{\mathrm{k}} \mathrm{f}(\mathrm{z})\right]^{\prime \prime}}{\mathrm{D}_{\lambda, \delta}^{\mathrm{k}} \mathrm{f}(\mathrm{z})^{\prime}}-\frac{\mathrm{z} \Phi^{\prime}\left[\mathrm{D}_{\lambda, \delta}^{\mathrm{k}} \mathrm{f}(\mathrm{z})\right]}{\Phi\left[\mathrm{D}_{\lambda, \delta}^{\mathrm{k}} \mathrm{f}(\mathrm{z})\right]}\right\}\right. \\
& \prec \frac{\alpha}{\mathrm{q}_{2}(\mathrm{z})}+\frac{\gamma \mathrm{zq}_{2}^{\prime}(\mathrm{z})}{\mathrm{q}_{2}(\mathrm{z})}
\end{aligned}
$$

holds, then:

$$
\mathrm{q}_{1}(\mathrm{z}) \prec \frac{\mathrm{z}\left[\mathrm{D}_{\lambda, \delta}^{\mathrm{k}} \mathrm{f}(\mathrm{z})\right]^{\prime}}{\Phi\left[\mathrm{D}_{\lambda, \delta}^{\mathrm{k}} \mathrm{f}(\mathrm{z})\right]} \prec \mathrm{q}_{2}(\mathrm{z})
$$

and $\mathrm{q}_{1}(\mathrm{z})$ is the best subordinant and $\mathrm{q}_{2}(\mathrm{z})$ is the best dominant.

\section{DISCUSSION}

The results describe the basic theory on certain applications of the field of differential subordination. The generalized differential operator obtained here 
covered the well known operators and many interesting results such as the coefficient estimates, distortion results and the sandwich theorem are found. Further many other results are yet to be studied.

\section{ACKNOWLEDGEMENT}

The work presented here was fully supported by SAGA GRANT:STGL-012-2006, Academy of Sciences, Malaysia.

\section{REFERENCES}

1. Al-Oboudi, F.M., 2004. On univalent functions defined by a generalized sălăgean operator. Int. J. Math. Math. Sci., 27: 1429-1436.

2. Al-Shaqsi, K. and M. Darus., 2008. An operator defined by convolution involving polylogarithms functions. J. Math. Stat., 4: 46-50.

3. Brickman, L., 1973. Ф-like analytic functions I. Bull. Am. Math. Soc., 79: 555-558.

4. Bulboaca, T., 2002. Classes of first-order differential superordinations. Demonstr. Math., 35: 287-292.

5. Miller, S.S. and P.T. Mocanu, 2003. Subordinants of differential superordinations. Complex Variables, 48: 815-826. DOI: $10.1080 / 02781070310001599322$.
6. Miller, S.S. and P.T. Mocanu, 2000. Differential Subordinations: Theory and Applications. Pure and Applied Mathematics. 1st Edn., CRC Press, Dekker, New York, pp: 480. ISBN-10: 0824700295.

7. Ruscheweyh, S., 1975. New criteria for univalent functions. Proc. Am. Math. Soc., 49: 109-115.

8. Sălăgean, G.S., 1983. Subclasses of univalent functions. Lecture Notes Math., 1013: 362-372. DOI: $10.1007 / \mathrm{BFb} 0066543$. 